\title{
Increased HLA-DR and decreased CD3 on human intestinal intraepithelial lymphocytes: evidence of activation?
}

\author{
M Abuzakouk, D Kelleher, C Feighery, C O’Farrelly
}

\begin{abstract}
Background-Activation of circulating $T$ lymphocytes results in expression of HLA-DR, interleukin-2 receptor (IL-2R), transferrin receptor ( $T r R)$, and decreased amounts of surface CD3.

Aim-To examine the activation status of human intestinal intraepithelial lymphocytes (IELs) by flow cytometry.

Patients and Methods-Duodenal biopsy specimens from 12 patients provided a source of intestinal lymphocytes. Dual colour analysis was used to compare activation marker expression by IELs with peripheral blood (PB) T lymphocytes from the same patients.

Results-All human IEL populations express HLA-DR and their density of expression is lower than on the small population of HLA-DR+ resting PB T lymphocytes (mean fluorescence intensity (MFI) 52.9, range 19.8-94.8 $v 152.6$ range, 49.1-320.3; $p<0.01$ ). The density of CD3 was significantly reduced on IELs (MFI 465.8, range 228.7-660), compared with $P B$ T lymphocytes $(756 \cdot 3$, range $444 \cdot 5-1573 \cdot 7$; p<0.009). IL-2R was not detected on IELs; nor were the activation markers, $T r R$, M21 C5, or M2 B3.
\end{abstract}

Conclusion-HLA-DR expression on IELs together with decreased CD3 expression suggest previous stimulation. However, the absence of additional markers of $T$ cell activation may reflect a unique pathway of activation.

(Gut 1996; 39: 396-400)

Department of Immunology M Abuzakouk C Feighery

Department of Gastroenterology, Dublin, Ireland D Kelleher

Education and Research Centre, St Vincent's Hospital, Dublin, Ireland C O'Farrelly

Correspondence to: Correspondence to: Education and Research Cducation and Research Centre, St Vincent's
Hospital, Dublin 4, Ireland. Hospital, Dublin 4, Ireland. 18 April 1996
Keywords: activation markers, intraepithelial lymphocytes, mean fluorescence intensity, major histocompatibility complex, peripheral blood.

Intraepithelial lymphocytes (IELs) are located between the epithelial cells of the gastrointestinal tract and are the first lymphocytes of the immune system to encounter exogenous antigens in the gut. However, their precise role in the gastrointestinal immune response is still unclear. In humans, IELs are predominantly CD8+ and most express the $\alpha \beta$ isoform of the $T$ cell receptor ( $\alpha \beta$ TCR), suggesting that they act as MHC class I restricted cytotoxic $T$ cells. ${ }^{1-3}$ By contrast with IELs from different animal species, human IELs show low selective spontaneous cellular cytotoxicity. ${ }^{4-6}$ Moreover, unlike peripheral blood (PB) CD8+ lymphocytes, the mitogen induced cytotoxic activity of human IELs is low and has been shown to have different target cell specificity. ${ }^{7}$ Most IELs express CD45 RO, ${ }^{12}$ a marker found on PB memory $T$ lymphocytes, ${ }^{8}$ suggesting that IELs are memory lymphocytes, which may have been previously primed with antigen, either in situ from luminal origin or in the process of trafficking to the gastrointestinal tract.

Previously, phenotypic studies of human IELs have relied almost exlusively on immunofluorescent and immunohistological examination of tissue sections. ${ }^{9-14}$ Using these techniques, quantification of subpopulations of cells and of surface marker expression has been problematic. With the development of flow cytometry and effective methods of tissue dispersal, it is now possible to identify and count populations of cells expressing two or more markers and to investigate the density of their expression. Using this approach, analysis of IELs in single cell suspensions prepared from biopsy specimens of the small intestine has disclosed several discrete subpopulations. ${ }^{2} 15$

Classically, $\mathrm{T}$ lymphocyte activation by exposure to antigens is characterised by changes in surface marker expression, including decreased CD3 and CD4 density accompanied by increased expression of HLADR, interleukin-2 receptor (IL-2R) and transferrin receptor (TrR). ${ }^{16-19}$ Expression of the recently described proliferation and activation associated antigens M21 C5 and M2 B3 is also known to occur on activated $T$ lymphocytes when stimulated in vitro by $T$ cell mitogens. ${ }^{20}{ }^{21}$ However, all these studies were performed exclusively on circulating lymphocytes. In this study, we used these features to examine by two colour flow cytometry the activation status of human intestinal IELs obtained from duodenal biopsy specimens. These results were compared with PB T lymphocytes from the same patients.

The study was approved by the ethics committee of the Federated Dublin Voluntary Hospitals, Ireland.

\section{Methods}

PATIENTS

Seven women and five men with mean age 45 (range 27-79) being investigated for upper 
gastrointestinal symptoms were studied. Disease of the small intestine was excluded at endoscopy and by routine histology of duodenal biopsy specimens in all patients.

\section{INTESTINAL TISSUE}

Six biopsy specimens were obtained by endoscopy from the second part of the duodenum from each patient. Two were placed in $10 \%$ formalin and sent for routine histology. The remaining four biopsy specimens were placed in ion free (calcium and magnesium free) Hank's balanced salt solution (Gibco) supplemented with antibiotics $(100 \mathrm{U} / \mathrm{ml}$ penicillin, $100 \mu \mathrm{g} / \mathrm{ml}$ streptomycin) and $5 \%$ fetal calf serum (FCS) to be used for flow cytometry.

PREPARATION OF SINGLE CELL SUSPENSIONS FROM DUODENAL BIOPSY SPECIMENS SUITABLE FOR FLOW CYTOMETRY

Four dueodenal biopsy specimens were used to prepare single cell suspensions suitable for flow cytometric analysis. The epithelial layer was removed with $1 \mathrm{mM}$ EDTA (Analar, BDH chemicals Ltd, Poole, England) and $1 \mathrm{mM}$ dithiothreitol (DDT, Sigma). After continuous agitation for one hour at $37^{\circ} \mathrm{C}$, the single cell suspension was pelleted from the supernatant and washed once with $5 \mathrm{ml}$ RPMI-1640 medium (Gibco, Life Technologies Ltd, Paisley, Scotland) supplemented with antibiotics and $10 \%$ FCS. To release lamina propria mononuclear cells, the remaining tissue was washed three times with RPMI1640 medium, disrupted with a scalpel, and treated with collagenase (type $1 \mathrm{~A}$, Sigma; 128 U/ml RPMI-1640 medium) for three hours with continuous agitation at $37^{\circ} \mathrm{C}$. The crude cell suspension was allowed to stand for five minutes to permit sedimentation of debris. Cells from the supernatant were washed twice with RPMI-1640 medium. Epithelial and lamina propria cell suspensions were resuspended in $1 \mathrm{ml}$ phosphate buffered saline (PBS) containing $1 \%$ bovine serum albumin (BSA) and $0.02 \%$ sodium azide and were kept on ice until stained.

PREPARATION OF PERIPHERAL BLOOD

MONONUCLEAR CELLS (PBMCs)

Heparinised venous blood $(10 \mathrm{ml})$ was also collected at the time of endoscopy from each patient. The PBMCs were obtained by FicollHypaque density gradient centrifugation at $400 \mathrm{~g}$ for 30 minutes (Lymphoprep Nycomed Pharma AS, Oslo, Norway) then washed twice with Hanks's balanced salt solution supplemented with HEPES buffer solution (Life Technologies Ltd, Paisley, Scotland) and antibiotics. Cell pellets were resuspended in $2 \mathrm{ml}$ PBS containing $1 \%$ BSA and $0.02 \%$ sodium azide and were kept on ice until stained. PB T lymphocytes from five controls were studied for surface marker expression before and after the treatment with EDTA and DDT.
CELL YIELD AND VIABILITY

Cell counts and viability were determined with ethidium bromide/acridine orange.

TWO COLOUR IMMUNOFLUORESCENCE

STAINING OF CELL PREPARATIONS

\section{Direct labelling}

Five microlitres of each monoclonal reagent were added to $100 \mu \mathrm{l}$ cell suspension $\left(1 \times 10^{5}\right.$ cells), incubated at room temperature for 10 minutes in the dark, and then washed twice with PBS, $1 \%$ BSA, and $0.02 \%$ sodium azide.

\section{Indirect labelling: two colour fluorescence for unconjugated monoclonal antibodies}

For unlabelled antibodies, after the second wash, $50 \mu \mathrm{l}$ 1:40 dilution FITC conjugated goat antimouse antibody was added; incubation and washing steps were repeated. Then $100 \mu 1$ 1:25 dilution of normal mouse serum was added to prevent the non-specific binding of the second antibody to the label. After repeating the incubation and the washing steps, the final phycoerythrin conjugated monoclonal antibody was added. Incubation and washing procedures were repeated as described. Cell pellets were resuspended in $750 \mu 10.5 \%$ paraformaldehyde in buffer and stored in the dark at $4^{\circ} \mathrm{C}$. Analysis was carried out within 15 hours of staining.

\section{Antibodies}

For assessing the activation status of $\mathrm{T}$ lymphocyte populations, the following monoclonal antibodies were used; phycoerythrin (PE) labelled anti-CD3, fluorescein isothiocyanate (FITC) labelled anti-IL-2R, Simultest anti-CD3 FITC labelled/anti-HLA-DR PE labelled, unlabelled anti-DP, and anti-DQ, all purchased from Becton Dickinson, Belgium. Monoclonal antibodies to transferrin receptor (L1B1), M21 C5, and M2 B3 antigens (gifts from Dr MB Omary, Stanford University, Palo Alto, CA, USA) were unlabelled.

TWO COLOUR FLOW CYTOMETRY

Flow cytometric acquisition and analysis were performed with a FACScan (Becton Dickinson, Belgium). The cell suspensions were initially visualised in the forward scatter/ side scatter (FSC-SSC) profile. A CD3 stained population was gated in FL1-FL2 and localised with FSC-SSC. A gate was drawn around the CD3+ population and used therafter. This gate excludes most epithelial cells and debris from the analysis. Acquisition of intestinal cell suspensions and PB samples from the same patient occurred on the same day. The FACScan settings were standardised regularly with flow cytometric beads (Calibrite, Becton Dickinson). Control reagent (FITC labelled IgG1 and PE labelled IgG2, Becton Dickinson) was used to exclude non-specific binding and to determine the optimum setting of fluorescence quadrants. Results were 


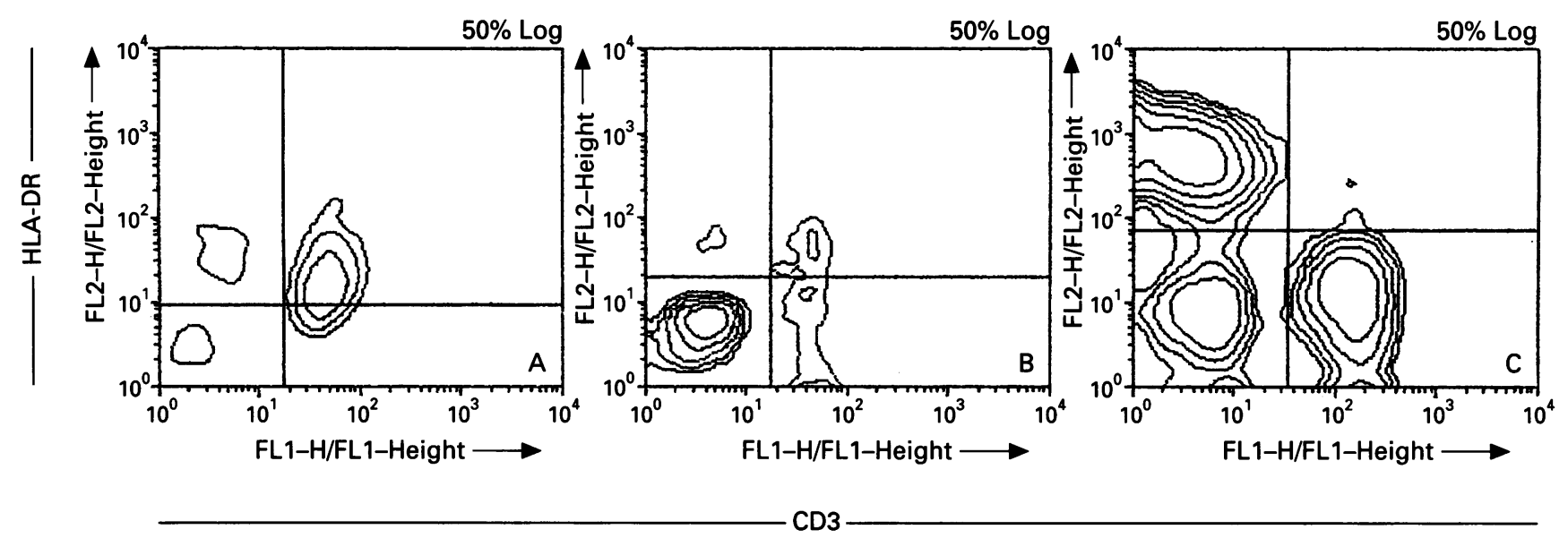

Figure 1: Two colour FACS profile showing (A) HLA-DR expression on intraepithelial lymphocytes, (B) lamina propria lymphocytes, and (C) PB lymphocytes. By contrast with lamina propria and PB T lymphocytes, IELs segregated as a single cell population with respect to DR expression. Quadrants were set with the use of background levels for FITC/PE conjugated mouse immunoglobin.

expressed as a percentage of total CD3+ cells calculated from quadrants for each tube. Density of surface markers was expressed as the mean fluorescence intensity (MFI); MFI was calculated as: MFI of cells stained with the specific monoclonal antibody - MFI of the same cells stained with the control monoclonal antibody.

\section{STATISTICS}

Data were analysed by Student's $t$ test. Results were considered significant at $\mathrm{p}<0.05$.

\section{Results}

CELL YIELD AND VIABILITY

Using four duodenal biopsy specimens, yields of epithelial layer cells ranged from $1.3 \times 10^{6}$ to $4.3 \times 10^{6}$ (mean $3.2 \times 10^{6}$ ); IELs accounted for $0.8 \times 10^{6}$ (range $0.5-1.5 \times 10^{6}$ ) cells; the remaining were enterocytes (mean $2.4 \times 10^{6}$, range $1 \cdot 8-3 \cdot 1 \times 10^{6}$ cells). In the lamina propria, the mean lymphocyte yield was $0.6 \times 10^{6}$ (range $0.1-1.8 \times 10^{6}$ ) cells. Cell viability in all cases exceeded $90 \%$.

ACTIVATION MARKER EXPRESSION BY IELS AND PBT LYMPHOCYTES

Flow cytometric analysis showed the IELs segregated as a single cell population with respect to DR expression. Expression was low with partial overlap with the background in about $25 \%$ of cells. By contrast lamina propria and PB lymphocytes segregated into two discrete populations with respect to DR positivity and negativity (Fig 1). Over one third of lamina propria lymphocytes (mean $32 \%$, range $22-46 \%$ ) and only $2 \cdot 8 \%$ (range $1 \cdot 3-5 \cdot 7 \%$ ) of PB T lymphocytes taken from the same patients expressed DR. However, the density of expression of HLA-DR was lower on the overall IEL population than on the small population of HLA-DR positive PB T lymphocytes (MFI of positive cells: $52 \cdot 9$, range $19 \cdot 8-94 \cdot 8 v 152 \cdot 6$, range $49 \cdot 1-320 \cdot 3 ; \mathrm{p}<0 \cdot 01$ )

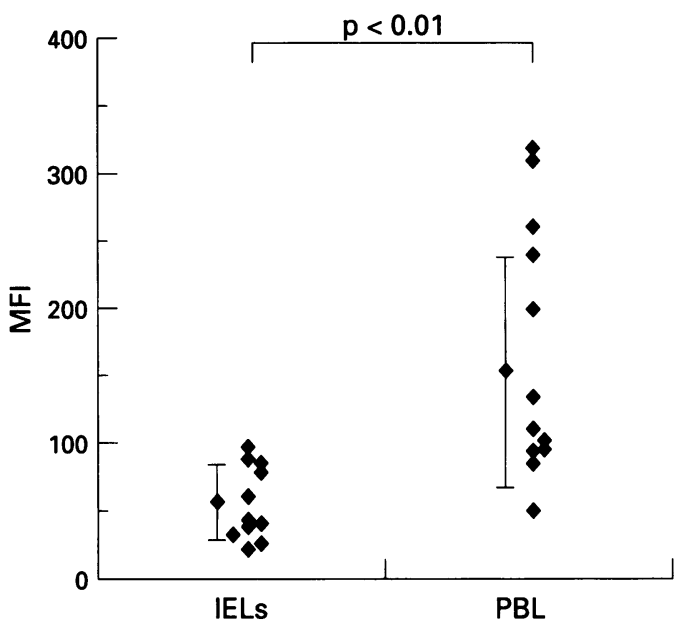

Figure 2: Mean fluorescence intensity (MFI) of HLA-DR expression on intraepithelial lymphocytes (IELS) and on the population $(1.3 \%-5 \cdot 7 \%)$ of HLA-DR positive PB T lymphocytes (PBL). Bars represent mean (SD).

respectively (Fig 2). The expression of DP and DQ was detected in $1 \%-4 \%$ and in $<1 \%$ of IELs respectively. In PB, $1 \%-6 \%$ and $<2 \%$ of CD3+ lymphocytes expressed DP and DQ respectively. The density of expression of the $\mathrm{T}$ cell surface marker $\mathrm{CD} 3$ was significantly reduced on IELs (465.8, range 228.7-660), compared with PB T lymphocytes (756.3, range 444.5-1573.7; $<<0.009)$ from the same patients (Fig 3). The expression of the activation marker IL-2R was not detected on IELs but was detected on a small population $(1 \%-2 \cdot 5 \%)$ of PB T lymphocytes. The IELs did not express the activation markers transferrin receptor, M21 C5, or M2 B3. Similarly, these markers were detected in $<1 \%$ of PB T lymphocytes. The expression of $D Q$, DP, IL-2R, transferrin receptor, M21 C5, and M2 B3 was not examined on lamina propria lymphocytes.

To ensure that decreased expression of HLA-DR and CD3 on IELs was not due to the effect of chelating and reducing agents used to obtain the epithelial layer from the duodenal biopsy specimens, PB lymphocytes were 


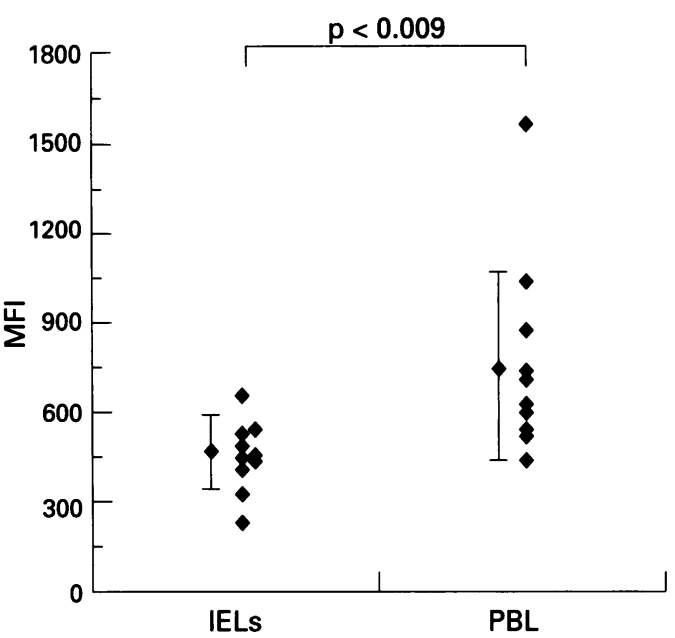

Figure 3: Mean fluorescence intensity (MFI) of $\mathrm{CD} 3$ expression on intraepithelial lymphocytes (IELs) and on peripheral blood T lymphocytes (PBLs). Bars represent mean $(S D)$.

treated with the same reagents and no significant effect on the expression of these markers was found. Moreover, the intensity of expression of CD8, $\alpha \beta$, and $\gamma \delta$ T cell receptors was similar on IELs and PB $\mathrm{T}$ lymphocytes obtained from the same patients (data not shown). Recently we have shown that the isolation procedure of lamina propria lymphocytes altered the density of expression of all surface markers examined in this study. ${ }^{22}$ Thus the intensity of expression lamina propria lymphocyte surface markers cannot be compared with IELs and PB T lymphocytes by this approach.

\section{Discussion}

In this study, flow cytometric analysis clearly shows specific DR expression by human IELs. Previously, HLA-DR expression in the small intestine was examined by double labelled immunofluorescent staining of histological sections. Although strong DR expression by enterocytes was detected, DR expression by IELs was not found with this approach. ${ }^{10} 142324$ Immunohistological techniques may yield equivocal results if the antigen of interest is expressed at a low density on cells surrounded by high expressing populations. Therefore, failure of previous immunohistological and immunofluorescence techniques to detect the expression of HLADR molecules on the surface of intestinal IELs could be due to a detection system that was inadequately sensitive for the lymphocyte subpopulations residing in the epithelial layer. With the development of flow cytometry, it is now possible to detect different low levels of surface marker expression on mixed cell subpopulations. Thus two colour immunofluorescent staining has recently shown that most CD8+ IELs express low levels of CD $5^{24}$ whereas immunohistological analysis show CD5 on only $50 \%$ of CD8+ IELs. ${ }^{123} 24$ Our studies confirm that flow cytometry is a more sensitive technique for showing low levels of surface marker expression; they show DR expression on all IELs that appeared negative for this marker on previous immunohistological staining.

Classic in vitro models have shown that $\mathrm{CD} 3$ expression decreases on $T$ cell activation ${ }^{18} 19$ whereas class II molecules are upregulated. In our study, DR expression by human IELs in the presence of decreased expression of CD3 suggests that these cells are activated. A previous study indicated that CD3 levels were similar on human IELs and PB CD8 T lymphocytes. ${ }^{25}$ However, it was not clear if the intensity of CD3 on IELs was compared with PB T lymphocytes from the same patients at the same time. In this study, we detected a significant diminution of $\mathrm{CD} 3$ expression on the surface of all IELs in comparison with PB $T$ lymphocytes from the same patients when paired sample analysis was carried out. This finding could suggest difference in cellular maturation or in the activation state of circulating $T$ lymphocytes and gut resident IELs in vivo. Activation responses of IELs and PB lymphocytes differ-for example, PHA activation results in reduced expression of LFA-1 on IELs whereas it upregulates LFA-1 on PB lymphocytes. ${ }^{26}$ Current studies in progress show significant differences in the response of surface molecules to cytokine activation in IELs $v$ PB lymphocytes.

An additional marker of classic $T$ cell activation is the expression of IL-2R, which is normally expressed early in the process of $T$ cell activation via the TCR. Studies of human IELs have shown that they produce IL-2 without the expression of the IL-2R when stimulated in vitro with $\mathrm{T}$ cell mitogens and that their proliferative response increased dramatically through stimulation of the CD2 receptor. $^{25-27}$ In this study, although a small percentage of PB T lymphocytes expressed IL$2 R$, IELs from the same patients had no detectable IL-2R expression. These results are in agreement with recently published work. ${ }^{28}$ Furthermore, the lack of expression of other proliferation and activation associated antigens including $\mathrm{TrR}, \mathrm{M} 21 \mathrm{C} 5$, and M2 B3 suggest that these gut lymphocytes may not be activated in the conventional sense. However, IELs express CD45 RO, which is present on memory $T$ lymphocytes. ${ }^{128}$ Hence, it is possible that these cells may have encountered antigens at a prior stage either in migration or during maturation, but have not responded in a classic fashion.

Our results emphasise the differences between lymphocytes located in the intestinal epithelium and those found in the circulation. Animal studies have shown that the expression of class II antigens by $\mathrm{T}$ cells is dependent on their location. ${ }^{29}$ In this study, the expression of HLA-DR by IELs but only a small percentage of PB T lymphocytes confirms the importance of local environment in influencing lymphocyte phenotype and thus function. Lymphocytes resident in the rheumatoid synovium, have also been reported to express HLA-DR in the absence of conventional activation markers. ${ }^{30}$ It is unclear whether class II molecules on $\mathrm{T}$ cells are functional although there is evidence 
that $T$ cells that have been induced to express HLA-DR are capable of presenting antigen to other $T$ cells and may also have immunoregulatory properties. ${ }^{31-33}$ It is possible that peptides produced by enterocytes could bind to DR on adjacent IELs, which could activate CD4+ IELs. Hence, the finding that virtually all IELs express HLA-DR suggests that these lymphocytes may have a discrete role, possibly in antigen presentation or immunoregulation. This may reflect their position within the gastrointestinal tract with continuous exposure to luminal antigens or a unique origin - there is evidence to suggest that human IELs can differentiate locally. ${ }^{15}$

During this study, MA was supported by a grant from the Ministry of Scientific Research, Libya. CO'F was supported by the Arthritis Foundation of Ireland and the Health Research Board of Ireland. DK is a Wellcome Senior Fellow in Clinical Science.

1 Brandtzaeg $P$, Bosnes V, Halstensen TS, Scott $H$, Sollid LM, Valnes KN. T lymphocytes in human gut epithelium preferentially express the $\alpha \beta$ antigen receptor and are often CD45/UCHL-1 positive. Scand $₹$ Immunol 1989; 30: 123-30.

2 Jarry A, Cerf-Bensussan N, Brousse N, Selz F, Guy-Grand D. Subsets of CD3+ (T-cell receptor $\alpha \beta$ or $\gamma \delta$ ) and CD3lymphocytes isolated from normal human gut epithelium display phenotypical features different from their counterparts in peripheral blood. Eur F Immunol 1990; 20: 1097-103.

3 Cerf-Bensussan N, Guy-Grand D, Griscelli C. Intraepithelial lymphocytes of human gut: isolation, characterisation and study of natural killer activity. Gut 1985; 26: 81-8.

4 Taunk J, Roberts AI, Ebert EC. Spontaneous cytotoxicity of human intraepithelial lymphocytes against epithelial cell tumours. Gastroenterology 1992; 102: 69-75.

5 Arnaud-Battandier F, Bundy BM, O'Neill M, Bienenstock J, Nelson DL. Cytotoxic activities of gut mucosal lymphoid cells in guinea pigs. F Immunol 1978; 121: 1059-65.

6 Tagliabue A, Luini W, Soldateschi D, Boraschi D. Natural killer activity of gut mucosal lymphoid cells in mice. Eur f Immunol 1981; 11: 919-26.

7 Ebert EC. Intraepithelial lymphocytes: interferon-gamma production and suppressor/cytotoxic activities. Clin Exp Immunol 1990; 82: 81-5.

8 Sanders ME, Makgoba MW, Shaw S. Human naive and memory $T$ cells: reinterpretation of helper-inducer and suppresser-inducer subsets. Immunol Today 1988; 9: $195-9$.

9 Kelly J, O'Farrelly C, O'Mahony C, Weir DG, Feighery C. Immunoperoxidase demonstration of the cellular composition of the normal and coeliac small bowel. Clin Exp Immunol 1987; 68: 177-88.

10 Malizia G, Trejdosiewicz LK, Wood GM, Howdle PD, Janossy G, Losowsky MS. The microenvironment of coeliac disease: $\mathrm{T}$ cell phenotypes and expression of the T2'T blast' antigen by small bowel lymphocytes. Clin Exp Immunol 1985; 60: 437-46.

11 Scott H, Sollid LM, Fausa O, Brandtzaeg P, Thorsby E. Expression of major histocompatibility complex class II subregion products by jejunal epithelium in patients with coeliac disease. Scand f Immunol 1987; 26: 563-71.

12 Spencer J, MacDonald TT, Diss TC, Walker-Smith JA, Ciclitira PJ, Isaacson PG. Changes in intraepithelial lymphocyte subpopulations in coeliac disease and enteropathy associated $T$ cell lymphoma (malignant histiocytosis of the intestine). Gut 1989; 30: 339-46.
13 Holm K, Savilahti E, Koskimies S, Lipsanen V, Maki M Immunohistochemical changes in the jejunum in first degree relatives of patients with coeliac disease and the coeliac disease marker DQ genes. HLA class II antigen expression, Interleukine-2 receptor positive cells and dividing crypt cells. Gut 1994; 35: 55-60.

14 Selby WS, Janossy G, Bofill M, Jewell DP. Lymphocyte subpopulations in the human small intestine. The findings in normal mucosa and in the mucosa of patients with adult coeliac disease. Clin Exp Immunol 1983; 52: 219-28.

15 Lynch S, Kelleher D, Feighery C, Weir DG, O'Farrelly C. Flow cytometric analysis of intraepithelial lymphocytes Fow cytometric analysis of intraepithelial lymphocytes from small intestinal biopsies reveals populations of Hepatol 1993; 5: 907-12.

16 Cantrell DA, Smith KA. Transient expression of interleukin 2 receptor. Consequences for T cell growth. $\mathcal{f} \operatorname{Exp~Med}$ 1983; 158: 1895-911.

17 Cotner T, Williams JM, Christenson L, Shapiro HM, Strom TB, Strominger J. Simultaneous flow cytometric analysi of human $T$ cell activation antigen expression and DNA content. $\mathcal{F}$ Exp Med 1983; 157: 461-72.

18 Matsui $Y$. Increased density of class I major histocompatibility complex antigens and decreased density of $T$ cell differentiation antigens in the early stages of T-cell activation. Hum Immunol 1987; 18: 123-33.

19 Weyand CM, Goronzy J, Fathman CG. Modulation of CD4 by antigenic activation. $\mathcal{F}$ Immunol 1987; 138: 1351-4.

20 Omary MB, Kelleher D, Kagnoff MF. An 80 to 85 Omary MB, Kelleher D, Kagnoff MF. An 80 to 85
kilodalton human phosphoglycoprotein associated with cell activation. F Immunol 1988; 141: 3492-7.

21 Omary MB, DeGrandpre L, Varki NM, Kagnoff MF. A tyrosine sulfated human glycoprotein with an unusual cel distribution. Mol Immunol 1992; 29: 9-19.

22 Abuzakouk M, Feighery C, O'Farrelly C. Collagenase and dispase enzymes disrupt lymphocyte surface molecules. $\mathcal{F}$ Immunol Methods 1996; 194: 211-6.

23 Trejdosiewicz LK, Badr-el-Din S, Smart CJ, Malizia G Oakes DJ, Heatley RV, Losowsky MS. Colonic mucosal $\mathrm{T}$ lymphocytes in ulcerative colitis: expression of CD7 antigen in relation to MHC class II (HLA-D) antigens. antigen in relation to $M H C$ class
Dig Dis $S c i$ 1989; 34: $1449-56$.

24 Trejdosiewicz LK. Intestinal intraepithelial lymphocytes and lymphoepithelial interactions in the human gastrointestinal mucosa. Immunol Lett 1992; 32: 13-20.

25 Ebert EC. Proliferative responses of human intraepithelia lymphocytes to various T-cell stimuli. Gastroenterology 1989; 97: 1372-81.

26 Kelleher D, Murphy A, Lynch S, O'Farrelly C. Adhesion molecules utilized in binding of intraepithelia lymphocytes to human enterocytes. Eur 7 Immunol 1994; 24: 1013-6.

27 Ebert EC. Sheep red blood cells enhance T-lymphocyte proliferation. Clin Immunol Immunopathol 1985; 37: 203-12.

28 Halstensen TS, Brandtzaeg P. Activated T lymphocytes in the celiac lesion:non-proliferative activation (CD25) of CD4 $+\alpha / \beta$ cells in the lamina propria but proliferation (Ki-67) of $\alpha / \beta$ and $\gamma / \delta$ cells in the epithelium. Eur $\mathcal{F}$ Immunol 1993; 23: 505-10.

29 Dutia BM, MacConnell I, Bird K, Keating P, Hopkins J. Patterns of major histocompatibility complex class II expression on $\mathrm{T}$ cell subsets in different immunological
compartments 1 . Expression on resting $\mathrm{T}$ cells. Eur $\mathcal{f}$ Immunol 1993; 23: 2882-8.

30 Cush JJ, Lipsky PE. Phenotypic analysis of synovial tissue and peripheral blood lymphocytes isolated from patients
with rheumatoid arthritis. Arthritis Rheum 1988; 31: with rhe 8 .

31 Russo C, Indiveri F, Quaranta V, Molinaro GA, Pellegrino MA, Ferrone S. Stimulation of human T lymphocytes by PHA-activated autologous T lymphocytes: Analysis of the role of Ia-like antigen with monoclonal antibodies. Immunogenetics 1981; 12: 267-74.

32 Brown MF, Cook RG, Van M, Rich RR. Cloned human T cells synthesise Ia molecules and can function as antigen presenting cells. Hum Immunol 1984; 11: 219-28.

33 Triebel F, De Roquefeuil S, Blanc C, Charron DJ, Debre P. Expression of MHC class II and Tac antigens on IL2activated human $T$ cell clones that can stimulate in MLR, AMLR, PLT and can present antigen. Hum Immunol 1986; 15: 302-15. 